Arch. Math. 88 (2007), 344-348

(C) 2007 Birkhäuser Verlag Basel/Switzerland

0003/889X/040344-5, published online 2007-03-14

DOI 10.1007/s00013-006-1987-0

Archiv der Mathematik

\title{
Completeness and compactness for varieties over a local field
}

\author{
Oliver LORSCHEID
}

\begin{abstract}
This paper shows that for a local field $K$, a subfield $k \subset K$ and a variety $X$ over $k, X$ is complete if and only if for every finite field extension $K^{\prime} \mid K$, the set $X\left(K^{\prime}\right)$ is compact in its strong topology.
\end{abstract}

Mathematics Subject Classification (2000). Primary 11G25; Secondary 14A10.

Keywords. Complete variety, local field, strong topology, compact variety.

1. Introduction. The main statement Theorem (1.1) of this article is a fact wellknown to experts, but which apparently misses a proof in the literature. The complex case is proven in [4, I §10]. For non-archimedean local fields, one can study rigid analysis as introduced by Tate and further developed by Remmert, Gerritzen, Kiehl, and others, which perhaps might lead to an analytic proof of the theorem. In the present paper however, we do not use methods from nonarchimedean analysis, but give instead an algebraic proof.

We define a local field to be a locally compact topological field. A topological field is a field with a non-discrete topology such that the field operations are continuous and points are closed. In [5, I §2-4] these fields were shown to be isomorphic and homeomorphic to the finite extensions of $\mathbb{R}, \mathbb{Q}_{p}$ or $\mathbb{F}_{p}((X))$ for some prime $p$.

Theorem 1.1. Let $K$ be a local field, $k \subset K$ a subfield and $X$ a variety over $k$. Then $X$ is complete if and only if for every finite extension $K^{\prime} \mid K$ of fields $X\left(K^{\prime}\right)$ is compact in its strong topology.

The author likes to thank Florian Pop, Jakob Stix, Stefan Wewers, Gunther Cornelissen and his own parents for their support. 
A pre-variety $X$ over a field $k$ is a geometrically reduced scheme of finite type over $k$, not necessarily irreducible. For a field extension $K \mid k$, the $K$-rational points of $X$ are defined to be the set $X(K)=\operatorname{Hom}_{k}(\operatorname{Spec} K, X)=\operatorname{Hom}_{K}$ (Spec $K, X \otimes_{k} K$ ) and can be viewed as a subset of the closed points of $X \otimes_{k} K$.

For every topological field $k$, we endow the sets of $k$-rational points of prevarieties over $k$ with a topology by requiring the following properties:

(i) $k \rightarrow \mathbb{A}_{k}^{1}(k)$ is a homeomorphism,

(ii) $(X \times Y)(k)=X(k) \times Y(k)$ has the product topology of $X(k)$ and $Y(k)$, and

(iii) for $Z \hookrightarrow X$ locally closed, $Z(k)$ has the subspace topology of $X(k)$.

There is a unique way of defining a topological structure like this and we will call it the strong topology on the pre-varieties over $k$. We remark that the strong topology is stronger than the Zariski topology. In the following, properties of spaces or maps in the strong topology are marked with the adverb "strongly", such as "strongly closed" for "closed in the strong topology".

If we have a morphism $\varphi: X \rightarrow Y$ of pre-varieties over $k$, then $\varphi(k): X(k) \rightarrow$ $Y(k)$ becomes strongly continuous. Similarly, if we have a pre-variety $X$ over $k$ and a homomorphism $k \rightarrow k^{\prime}$ of topological fields which is a topological embedding, then the induced map $X(k) \rightarrow X\left(k^{\prime}\right)$ also is a topological embedding. This means that properties like open, closed or dense in the strong topology are stable under such field extensions. In particular, extensions of fields with absolute value are of this form. By definition of a variety, the $k$-rational points of a $k$-variety form a Hausdorff space.

To see that it is necessary to consider finite field extensions in theorem (1.1) look at the example $X=\operatorname{Spec} \mathbb{R}\left[X_{1}, X_{2}\right] /\left(X_{1}^{2}+X_{2}^{2}-1\right)$. Here $X(\mathbb{R}) \simeq \mathbb{S}^{1}$ is compact, but $X(\mathbb{C}) \simeq \mathbb{C}^{\times}$is not. Another example is to take an irreducible polynomial $f\left(X_{1}\right)$ of degree $n \geq 2$ over a local field $K$. Then $X=\operatorname{Spec} K\left[X_{1}, X_{2}\right] / f\left(X_{1}\right)$ has no $K$-rational points, hence $X(K)$ is compact. But for the splitting field $K^{\prime}=K(f)$, $X\left(K^{\prime}\right)$ is the union of $n$ affine spaces which certainly is not compact.

2. From Completeness to Compactness. In this section, $K$ denotes a local field and $|\cdot|$ its absolute value.

The key result for the proof that a complete variety $X$ over $K$ is compact in its strong topology is Chow's Lemma. A proof can be found in $[1,5.6 .1]$, also compare with $[4, \mathrm{I} \oint 10]$.

Theorem 2.1 (Chow's Lemma). Let $X$ be a reduced scheme that is proper and of finite type over a noetherian scheme $S$. Then there is a reduced projective scheme $Y$ of finite type over $S$, a surjective morphism $\varphi: Y \rightarrow X$ over $S$, and an open and dense subset $U \subset X$ such that the restriction $\varphi: \varphi^{-1}(U) \rightarrow U$ is an isomorphism.

Using the projection of the $(n+1)$-sphere to $\mathbb{P}^{n}(K)$, one sees that the $K$-rational points of projective space form a strongly compact set. For this, it is crucial that 
$K$ is locally compact. Therefore, strongly closed subsets of projective space are also strongly compact, and we obtain:

Lemma 2.2. For a projective variety $X$ over $K$, the set $X(K)$ is strongly compact.

Now we can conclude via Chow's Lemma:

Theorem 2.3. More generally, for a complete variety $X$ over $K$, the set $X(K)$ is strongly compact.

Proof. The proof is by induction with respect to $\operatorname{dim} X$.

For $\operatorname{dim} X=0, X(K)$ is a finite set and therefore compact.

For $\operatorname{dim} X>0$, by Chow's Lemma there is a projective variety $Y$ and a surjective morphism $\varphi: Y \rightarrow X$ as well as an open and dense subvariety $U \subset X$ such that $\varphi: V \rightarrow U$ is an isomorphism for $V=\varphi^{-1}(U)$.

We obtain the following diagram of sets and maps:

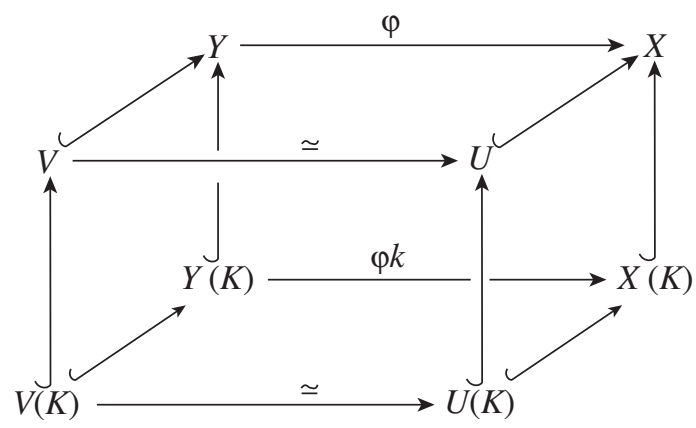

As an isomorphism between $V$ and $U, \varphi$ is a bijection between their $K$-rational points and $U(K)=U \cap X(K)$ is part of the image of $\varphi_{K}: Y(K) \rightarrow X(K)$.

Now the closed subvariety $Z=X-U$ has the same $K$-rational points as $X$ outside of $U$. Since $U \subset X$ is an open and dense subvariety, $\operatorname{dim} Z<\operatorname{dim} X$, and since $Z \rightarrow X$ is a closed subscheme, $Z$ is complete. Hence we can assume by induction that $Z(K)$ is strongly compact.

Finally, as the union of the two strongly compact sets $Z(K)$ and $\varphi_{K}(Y(K))$, $X(K)$ is itself strongly compact.

Now $X(K)=\operatorname{Hom}_{K}\left(K, X \otimes_{k} K\right)$ if $X$ is defined over a subfield $k \subset K$. Since completeness is stable under base extension, and keeping in mind that we can replace $K$ by any finite extension $K^{\prime}$, the first half of theorem (1.1) follows:

Corollary 2.4. If $X$ is a complete variety over $k \subset K$, then $X(K)$ is strongly compact. 
3. From Compactness to Completeness. To prove the reverse implication, we will make use of the following two theorems.

Theorem 3.1 (Implicit function theorem). Let $K$ be a complete field with absolute value ||$, X$ an $r$-dimensional variety over $K$ and $\varphi: X \rightarrow \mathbb{A}_{K}^{r}$ a morphism that is étale at a $K$-rational point $p \in X$ and maps $p$ to a $K$-rational point. Then $\varphi_{K}: X(K) \rightarrow \mathbb{A}_{K}^{r}(K)$ is a strong homeomorphism near $p$.

This theorem is a well-known statement which in the archimedean case is nothing else than the usual implicit function theorem and in the non-archimedean case results from the multidimensional Hensel's Lemma.

Theorem 3.2 (Nagata). Let $k$ be an arbitrary field. Every variety over $k$ can be embedded into a complete variety over $k$ as an open and dense subset.

A proof of this theorem can be found in [3].

Now it is possible to prove the other direction of theorem (1.1). This part of the proof holds for any field $K$ complete with respect to an absolute value. Let $k$ be a subfield of $K$.

Theorem 3.3. Let $X$ be a variety over $k$ such that for every finite extension $K^{\prime} \mid K$, $X\left(K^{\prime}\right)$ is strongly compact. Then $X$ is complete.

Proof. If $\operatorname{dim} X=0$, there is nothing to prove. Let $\operatorname{dim} X>0$.

By Nagata's theorem, $X$ can be embedded into a complete variety $Y$ over $k$ as an open and dense subset. If $X$ was not complete there would be a closed point $p \in Y-X$. But we will show that the existence of $p$ leads to a contradiction.

Since $X \neq Y$ implies $X \otimes k^{\prime} \neq Y \otimes k^{\prime}$ for every extension $k^{\prime} \mid k$, we can change the base field to prove the non-existence of the point $p$. Further, we can substitute $K$ by a finite extension $K^{\prime}$ without changing the validity of the conditions of the theorem. First, we assume that $X$ and $Y$ are varieties over $K$ and that $p$ is $K$-rational.

In an affine neighbourhood of $p$, we can find a curve through $p$ that intersects $X$ non-trivially. Let $C$ be its closure in $Y$, then $C \cap X$ is closed in $X$ and therefore $(C \cap X)\left(K^{\prime}\right)$ strongly compact for every $K^{\prime} \mid K$ finite. Replace $Y$ by $C$ and $X$ by $C \cap X$, then $Y-X$ has only finitely many points.

Since $X(K)$ is strongly compact, it is strongly closed in $Y(K)$. It follows that $p$ is a strongly discrete point as the complement of finitely many strongly closed points in the strongly open set $(Y-X)(K)$.

The normalisation $Z \rightarrow Y$ is a finite morphism, and therefore there are finitely many discrete points in the inverse image of $p$. Since the normalisation of a curve over an algebraically closed field is smooth, we can enlarge $K$ such that the normalisation of $Y$ is smooth. Hence we can assume that $Y$ is a normal and smooth curve. 
A smooth curve is locally étale over $\mathbb{A}_{K}^{1}$, compare with $[2$, III 10.4] and [4, III $\S 6 \mathrm{Thm} .1]$. This means that there is an open neighbourhood $U \subset Y$ of $p$ and an étale morphism $\varphi: U \rightarrow \mathbb{A}_{K}^{1}$. By another finite field extension, we can assume that not only $p$, but also $\varphi(p)$ is $K$-rational. Then the implicit function theorem (3.1) shows that there are homeomorphic strong neighbourhoods of $p$ and $\varphi(p)$. But $\varphi(p)$ cannot be strongly discrete since $\mathbb{A}_{K}^{1}(K) \cong K$ is non-discrete. Contradiction!

\section{References}

[1] A. Grothendieck and J. Dieudonné, Eléments de Géométrie Algébrique II. Publ. Math. IHES 8, (1961).

[2] R. Hartshorne, Algebraic Geometry. Springer-Verlag, New York 1977.

[3] H. NaGATA, Imbedding of an Abstract Variety in a Complete Variety. J. Math. Kyoto Univ. 2, 1-10 (1962).

[4] D. Mumford, The Red Book of Varieties and Schemes. second edition, SpringerVerlag, Berlin-Heidelberg 1999.

[5] A. WeIL, Basic Number Theory. Springer-Verlag, Berlin-Heidelberg-New York 1967.

OlIVER LORSCHEID, Department of Mathematics, Utrecht University, Budapestlaan 6, NL-3584 CD Utrecht, The Netherlands

e-mail: lorscheid@math.uu.nl

Received: 13 April 2006 